\title{
An unusual presentation of choriocarcinoma
}

Amishrita Lakku Rao*, Rama Sarala Devi

Department of Obstetrics and Gynecology, Kakatiya Medical College, Warangal, Telangana, India

Received: 24 January 2017

Revised: 22 February 2017

Accepted: 02 March 2017

\section{*Correspondence:}

Dr. Amishrita Lakku Rao,

E-mail: 1.amishrita@gmail.com

Copyright: () the author(s), publisher and licensee Medip Academy. This is an open-access article distributed under the terms of the Creative Commons Attribution Non-Commercial License, which permits unrestricted non-commercial use, distribution, and reproduction in any medium, provided the original work is properly cited.

\begin{abstract}
Choriocarcinoma is the most malignant growth arising from the uterine body. Incidence of choriocarcinoma following vesicular mole is $29-83 \%$. Diagnosis of choriocarcinoma is by histopathology only. In the present case 22year old woman with history of vesicular mole evacuation 8 months back suddenly landed in shock. During her irregular follow up, misleading ultrasonographic findings and clinical features delayed the diagnosis of advanced choriocarcinoma till it perforated through the uterus and patient landed in shock due to massive hemoperitoneum.

We report this case because of its unusual presentation which led to diagnostic dilemma and mismanagement.
\end{abstract}

Keywords: Beta hCG, Choriocarcinoma, Methotrexate, Quiescent GTD

\section{INTRODUCTION}

Choriocarcinoma is a rare tumour but it is the most malignant growth arising in the body of the uterus.50\% of cases follow evacuation of hydatiform mole $25 \%$ follow abortion and $20 \%$ follow full term pregnancy. $5 \%$ of cases follow extra uterine pregnancy. ${ }^{1}$ Choriocarcinoma continues to grow into the surrounding tissues until it perforates through the uterus. Hence, Choriocarcinoma is a diagnostic and therapeutic challenge with potential severe consequences if uterine perforation occurs along with massive intraperitoneal hemorrhage. This case is reported because of its unusual presentation which led to diagnostic dilemma and mismanagement.

\section{CASE REPORT}

A 22yr nulliparous woman was brought in a state of shock to the emergency outpatient in Government Maternity hospital Hanamkonda. History revealed suction and evacuation for molar pregnancy 8 months back. Following this the patient did not come to the hospital for follow up and did not use any contraception. Her menstrual cycle resumed for a month followed by 3 months amenorrhea. Urine pregnancy test (gravindex test) was positive. Patient developed spotting and consulted the local physician who advised a scan. Scan showed products in the cavity with features suggestive of incomplete abortion. She was advised to undergo evacuation but she did not comply and continued with the presumed pregnancy. Following this she developed sudden onset severe pain abdomen and collapse and was brought to our hospital in a state of shock. On examination she had severe pallor, cold clammy extremities, weak pulse, systolic blood pressure of $70 \mathrm{~mm}$ of $\mathrm{Hg}$ and diastolic blood pressure of $40 \mathrm{~mm}$ of $\mathrm{Hg}$. Respiratory rate was 26 per min. Auscultation revealed no abnormalities. Vesicular breath sounds and both heart sounds were heard. No adventitious sounds. There was mild distension of the abdomen with tenderness and guarding. Per abdominal examination revealed a firm mass corresponding to uterine size of 16 weeks. All the margins were well defined except the lower margin which was not within reach. No organomegaly was noted. Bowel sounds were heard. Per speculum examination 
showed a healthy cervix with altered blood coming through the os.

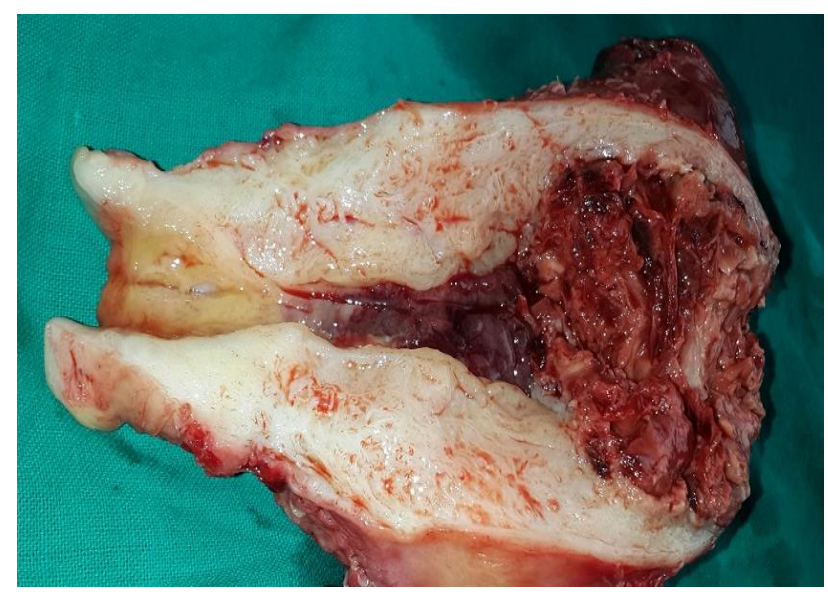

Figure 1: Cut section of the specimen.

On per vaginal examination uterus of 16 weeks sizewith cystic masses in both the fornices was found. Cervical movements were tender and the mass movements were transmitted to cervix. Fullness was noted in the pouch of Douglas. On per rectal examination rectal mucosa was free. Fullness in the pouch of Douglas was noted.

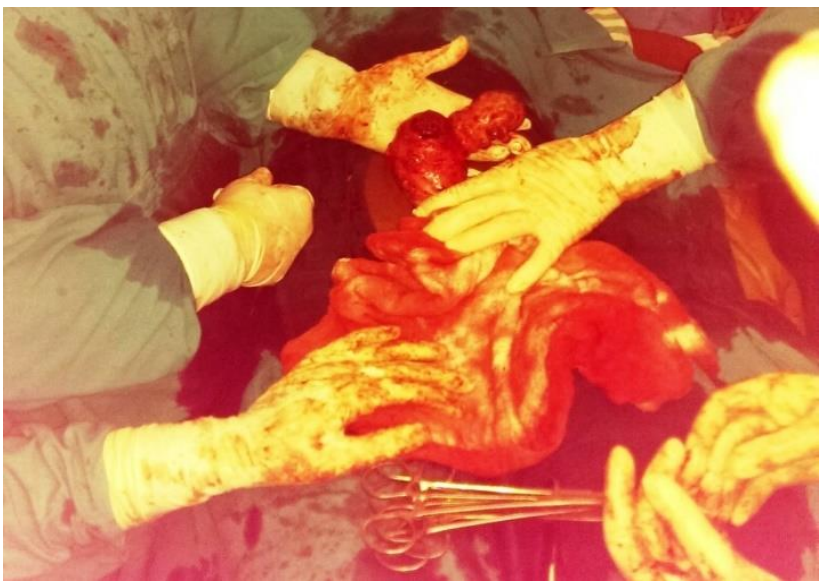

Figure 2: Intraoperative image.

\section{Haematological investigations revealed}

- Haemoglobin of $7 \mathrm{gm} \%$

- $\mathrm{O}^{+}$blood group

- Viral markers were non-reactive

- Blood urea and serum creatinine were within normal limits

- Urine for gravindex was strongly positive.

- Serum Beta hCG levels were found to be more than $1,00,000 \mathrm{mIU} / \mathrm{ml}$.

\section{Ultrasonography of the abdomen and pelvis revealed}

- Massive hemoperitoneum with features suggestive of invasive mole.
- Bilateral multilocular ovarian cysts of $6 \times 7 \mathrm{~cm}$.

- Liver and spleen was found to be normal.

Chest X-ray showed no lung deposits.

After treating the shock, 3 transfusions of compatible blood were done and patient was taken up for emergency laparotomy with 2 units of packed red cells and 2 units of fresh frozen plasma in hand. During laparotomy hemoperitoneum of 2 litres was found. Uterus was about 16 weeks size with a rent in the right cornua extending into the uterine vessels. Purple colored growth was seen through the rent with purple colored deposits on the right ovary right fallopian tube and on the posterior aspect of the uterus. Total abdominal hysterectomy with right salpingo oophorectomy was performed. Peritoneal wash was given and hemostasis secured. Abdomen was closed in layers and the specimen sent for histopathology. Cut section revealed purple color fungating growth near the fundus more towards the right side which is friable. Histopathological exami78nation revealed a well differentiated choriocarcinoma. In the post-operative period methotrexate was administered to the patient on day 1, 3, 5 and 7 with alternate folinic acid rescue. Postoperative recovery was uneventful. Suture removal was done on $7^{\text {th }}$ postoperative day and patient was referred to higher centre for chemotherapy.

\section{DISCUSSION}

The incidence of choriocarcinoma is in the order of $1: 50,000$ to $1: 70,000$ pregnancies in UK and USA. It is ten times more common in South East Asian women. ${ }^{1}$ Older women with high parity and belonging to a low socioeconomic group runs a high risk of developing this malignancy.

\section{Non-metastatic tumour}

It develops in about $15 \%$ of patients following evacuation of a complete hydatiform mole and infrequently after other gestations. Patient may present with

- Irregular vaginal bleeding.

- Theca lutein cysts.

- Uterine sub-involution or asymmetric enlargement.

- Persistently elevated beta HCG levels. ${ }^{2}$

The tumour may perforate the myometrium causing intra peritoneal bleeding or erode into uterine vessels. Bulky necrotic tumour may involve the uterine wall and become a nidus for infection presenting as purulent vaginal discharge with acute pelvic pain.

\section{Metastatic tumour}

It occurs in about $4 \%$ of cases following evacuation for complete mole but it is more often seen after non-molar pregnancies $^{2}$. Its tendency for early vascular invasion and perfusion by fragile vessels leads to widespread 
dissemination. Most common sites for metastases are lung $(80 \%)$, vagina $(30 \%)$, pelvis $(20 \%)$, liver $(10 \%)$ and brain $(10 \%) .^{2}$ The patient presents with features like hemoptysis, cough, chest pain, dyspnea or sometimes asymptomatic. Chest $\mathrm{x}$ ray may show an alveolar or snow storm pattern, discrete rounded densities (cannon ball appearance), pleural effusion or tumour emboli. Vaginal metastases may present with vaginal bleed or purulent discharge. They are highly vascular and bleed vigorously. They may be present in the fornices or suburethrally as purple coloured friable masses. Liver metastases presents with epigastric or right upper quadrant pain due to stretch on the hepatic capsule. It may also present as hepatic rupture with massive hemoperitoneum. Cerebral involvement usually presents along with vaginal or lung metastases. Patient generally presents with acute focal neurological deficit or convulsions. Think of choriocarcinoma if a young woman develops neurological symptoms with an1 year of abortion or pregnancy and estimate beta HCG levels in the CSF. ${ }^{1}$ Our patient presented with the non-metastatic tumour and there were no features suggestive of extrauterine involvement. Optimal evaluation of persistent gestational trophoblastic neoplasia requires a thorough assessment of the extent of spread before initiation of treatment. ${ }^{3,4}$

- Complete history and physical examination.

- Measurement of baseline serum beta hCG.

- Hepatic thyroid and renal function tests.

- Determination of baseline peripheral white blood cell and platelet count.

\section{Metastatic work up includes}

- Chest radiograph or CT scan.

- Ultrasonography or CT scan of the abdomen and pelvis.

- CT or MRI scan of the head.

An anatomic staging was proposed by FIGO (International federation of gynaecology and obstetrics) for comparison of data from various centres. ${ }^{4}$

\section{Figo staging of choriocarcinoma}

- $\quad$ Stage 1: persistently elevated beta HCG and disease confined to the uterus.

- $\quad$ Stage 2: tumour extending outside uterus but limited to genital structures.

- Stage 3: pulmonary metastases with or without uterine vaginal or pelvic involvement.

- Stage 4: advanced disease with brain liver kidney or gastrointestinal tract involvement.

Our patient was in stage 2 according to FIGO staging. WHO (World health organisation) proposed a prognostic scoring system which predicts the potential for resistance to chemotherapy. It is based on various factors such as age, antecedent pregnancy, interval between end of antecedent pregnancy and start of chemotherapy, beta HCG levels, blood group, largest tumour size, site and number of metastases and prior chemotherapy. Total score of $<7$ suggests low risk disease and $>7$ is high risk. ${ }^{2}$

Table 1: WHO prognostic scoring system for choriocarcinoma.

\begin{tabular}{|c|c|c|c|c|}
\hline Criteria & 0 & 1 & 2 & 4 \\
\hline Age (in years) & 39 years and below & >39years & & \\
\hline Antecedent pregnancy & Hydatiform mole & Abortion & Term pregnancy & \\
\hline $\begin{array}{l}\text { Interval between antecedent pregnancy } \\
\text { and start of chemotherapy }\end{array}$ & $<4$ months & 4-6 months & 7-12months & $>12$ months \\
\hline Human chorionic gonadotrophin(IU/L) & $<1000$ & $1000-10,000$ & $10,000-1,00,000$ & $>1,00,000$ \\
\hline $\mathrm{ABO}$ groups & & $\mathrm{O}$ or $\mathrm{A}$ & $\mathrm{B}$ or $\mathrm{AB}$ & \\
\hline Largest tumor including uterine $(\mathrm{cm})$ & $<3$ & $3-5$ & $>5$ & \\
\hline Site of metastases & & Spleen, kidney & GI tract & Brain, liver \\
\hline Number of metastases & & $1-3$ & $4-8$ & $>8$ \\
\hline Prior chemotherapy & & & 1 drug & 2 or more drugs \\
\hline
\end{tabular}

Our patient belonged to the low risk category with a total score of 6 .

\section{Management of low risk disease without metastases}

In a woman wishing to retain her fertility single agent chemotherapy is the preferred treatment with stage 1 disease. Methotrexate or Actinomycin D achieved similar remission rates but due to better toxicity profile for MTx -folinic acid regimen it is the preferred first line agent. It is given in dosage of $1 \mathrm{mg} / \mathrm{kg}$ body weight either as a 5day regimen or pulsatile weekly regimen. It can be given orally, intramuscular route or bolus intravenously. Folinic acid is administered on alternate days. Serum beta HCG level is measured weekly after each course of chemotherapy. Second course is withheld as long as the hCG level is falling progressively. It is started in the following situations: 
- If the hCG level plateaus or continues to rise for 3 consecutive weeks.

- If the decrease in hCG is less than $1 \log$ within 18days after the completion of first treatment. ${ }^{2}$

In a woman who does not wish to preserve fertility, completed her family with age above 35years hysterectomy with adjuvant single agent chemotherapy may be performed as the primary treatment.

\section{Low risk metastatic disease}

Pulmonary spread usually resolves with single agent chemotherapy. Occasionally thoracotomy may be required for a single focus not resolving with chemotherapy. Vaginal and pelvic metastases have a high remission rate with single agent chemotherapy and usually require combination chemotherapy.

\section{High risk disease}

All patients should be treated with primary intensive combination chemotherapy and selective use of radiation and surgery. Combination chemotherapy includes etoposide with MTx, Actinomycin D, cyclophosphamide and vincristine (EMA-CO regimen). Recently vinblastine, ifosfamide and paclitaxel have also been used successfully. ${ }^{2}$

\section{Quiescent gestational trophoblastic disease}

It is a syndrome involving benign or inactive gestational trophoblastic disease. It comprises solely or predominantly of highly differentiated syncitiotrophoblast cells. It has minimal extravillous or villous or cancer cell cytotrophoblast cells so lacks hyperglycosylated beta hCG the invasive signal. It commonly self resolves with disappearance of beta hCG over 6 months. Chemotherapy is ineffective as the cells are slow growing and it is advised to avoid chemotherapy in quiescent GTD. In $22 \%$ cases quiescent GTD preceded invasive disease. In these cases hyperglycosylated hCG became evident prior to significant rise in total hCG, which is the first evidence if invasive disease. Hence, it is critical to monitor hCG regularly in quiescent GTD. It is recommended that women with quiescent GTD be placed on oral contraceptive pills and avoid pregnancy atleast until beta hCG has been undetectable for 6 months. ${ }^{5}$

\section{Follow up}

All patients with low risk gestational trophoblastic neoplasia (stage 1 to stage 3 ) should undergo follow up with:
- Weekly measurement of hCG levels until they are normal for 3 consecutive weeks.

- Monthly measurement of hCG values until they are normal for 12 consecutive months.

- Effective contraception during the entire period of hormonal follow up. ${ }^{2}$

All patients with stage 4 disease should receive follow up with:

- Weekly determination of hCG levels until they are normal for 3 consecutive weeks.

- Monthly determination of hCG levels until they are negative for 24 consecutive months.

- Thereafter the patient should be followed up every 6months lifelong. ${ }^{2}$

\section{CONCLUSION}

Our patient belonged to low socio-economic status and resided in a tribal area. She was not aware of the seriousness of her condition and did not comply with the advice given by the local physician. She did not report to the hospital for follow up and did not use any contraception. Poor access to medical care, reluctance of the patient to seek treatment at tertiary care centre, financial constraints and lack of awareness together led to the disastrous consequence of uterine perforation and hysterectomy. This could have been avoided with proper counselling of the patient and follow up.

Funding: No funding sources

Conflict of interest: None declared

Ethical approval: Not required

\section{REFERENCES}

1. Howkins and Bourne Shaw's text book of gynaecology $16^{\text {th }}$ Edition. Chapter 39 Cancers of Endometrium, Uterus and Fallopian tube; 514-516.

2. Berek and Novak's Gynaecology $15^{\text {th }}$ Edition Chapter 39 Gestational Trophoblastic Disease Pages; 1467-1468,1471-1472,1474-1475.

3. Chemotherapy Research and Pract Volume 2011 Article ID http://dx.doi.org/10.1155/2011/ by Taymaa May Donald P Goldstein Ross S Berkowitz.

4. DC Dutta Text Book of Gynecology $7^{\text {th }}$ Edition. Chapter 24; 2015:302.

5. Cole La New Discoveries on biology and detection of Human Chorionic Gonadotrophin. Reprod Biol Endocrinol 2009;7:8.

Cite this article as: Rao LA, Devi SR. An unusual presentation of choriocarcinoma. Int J Reprod Contracept Obstet Gynecol 2017;6:1668-71. 